\title{
H 1504+65 - The Naked Stellar C/O Core of a Former Red Giant Observed with FUSE and Chandra
}

\author{
Klaus Werner, Thomas Rauch \\ Institut für Astronomie und Astrophysik, Univ. Tübingen, Sand 1, \\ D-72076 Tübingen, Germany \\ Martin A. Barstow \\ Department of Physics and Astronomy, University of Leicester, \\ University Road, Leicester LE1 $7 R H$, UK \\ Jeff W. Kruk \\ Department of Physics and Astronomy, Johns Hopkins University, \\ 3400 North Charles Street, Baltimore MD 21218, USA
}

\begin{abstract}
H} 1504+65$ is an extremely hot hydrogen-deficient white dwarf with an effective temperature close to $200000 \mathrm{~K}$. We present new FUV and soft X-ray spectra obtained with FUSE and Chandra, which confirm that $\mathrm{H} 1504+65$ has an atmosphere primarily composed of carbon and oxygen. The Chandra spectra show a wealth of absorption lines from highly ionized oxygen, neon and magnesium and suggest relatively high $\mathrm{Ne}$ and $\mathrm{Mg}$ abundances. This corroborates an earlier suggestion that $\mathrm{H} 1504+65$ represents a naked $\mathrm{C} / \mathrm{O}$ stellar core or even the $\mathrm{C} / \mathrm{O}$ envelope of an $\mathrm{O} / \mathrm{Ne} / \mathrm{Mg}$ white dwarf.
\end{abstract}

\section{Introduction}

H $1504+65$ is an optically faint blue star. It has been identified as the counterpart of a bright soft X-ray source (Nousek et al. 1986) that was discovered by an early X-ray survey (Nugent et al. 1983). Spectroscopically, the star is a member of the PG 1159 class, which comprises hot hydrogen-deficient post-AGB stars $\left(T_{\text {eff }}=75000-180000 \mathrm{~K}, \log g=5.5-8\right.$; Werner 2001). The PG 1159 stars are probably the outcome of a late helium-shell flash, a phenomenon that drives the currently observed fast evolutionary rates of three well-know objects (FG Sge, Sakurai's object, V605 Aql). A late helium-shell flash may occur in a post-AGB star or a white dwarf, and brings the star back onto the AGB. Flash induced mixing generates a $\mathrm{H}$-deficient surface layer and the subsequent second post-AGB evolution explains the existence of Wolf-Rayet central stars of planetary nebulae and their successors, the PG 1159 stars (see also Schönberner \& Jeffery page 173). Within the PG 1159 group H $1504+65$ is an extraordinary object, as it has been shown that $\mathrm{H} 1504+65$ is not only hydrogen-deficient but also helium-deficient. From optical spectra it was concluded that the atmosphere is primarily composed of carbon and oxygen, by equal amounts (Werner 


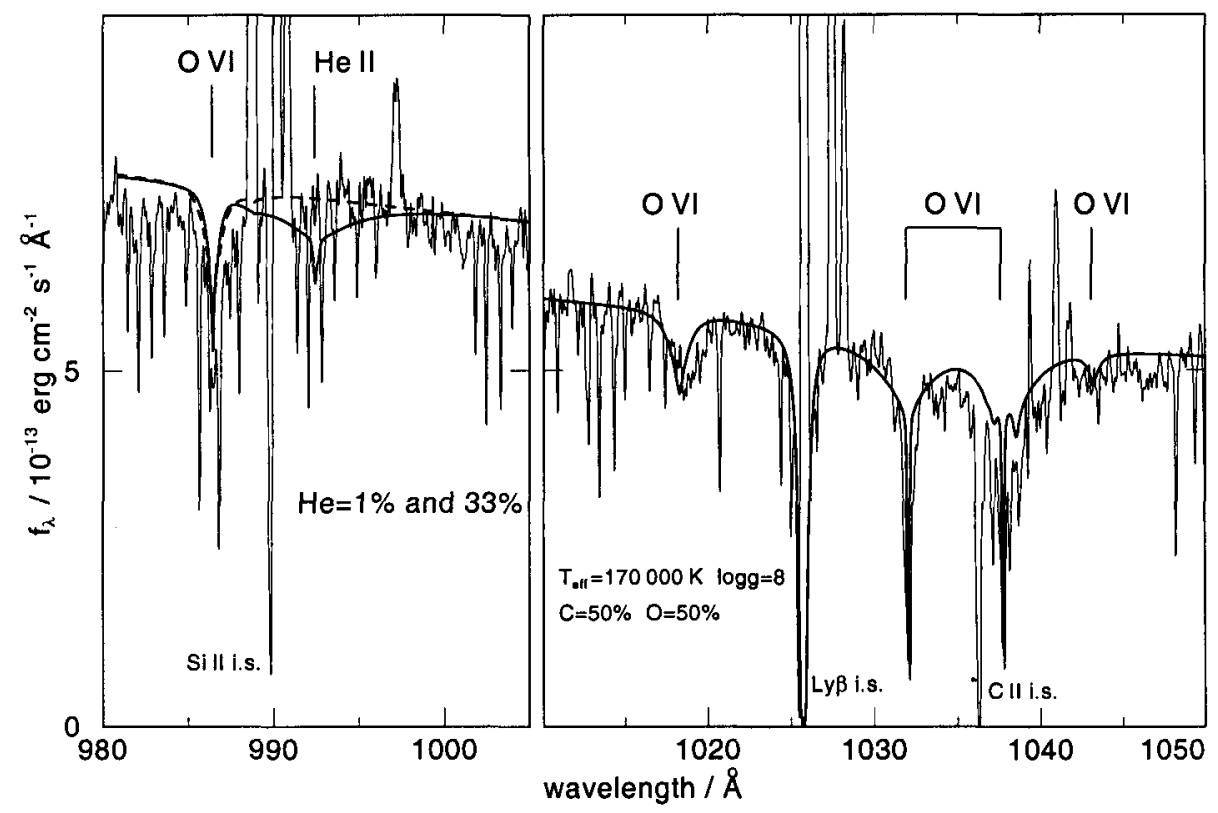

Figure 1. Details from the FUSE spectrum of H 1504+65. Left panel: The lack of the He II $(n=2 \rightarrow 7)$ absorption line confirms the Hedeficiency. Overplotted are two models with $33 \% \mathrm{He}$ and $1 \% \mathrm{He}$. Right panel: The OVI resonance doublet shows no signature of a stellar wind. Narrow absorption lines are of interstellar origin (mostly $\mathrm{H}_{2}$ ) and emission lines are from the geocorona. All spectra are smoothed

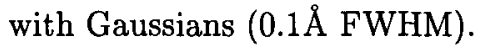

1991). Neon lines were detected in soft X-ray spectra taken with the EUVE satellite and in an optical-UV Keck spectrum and an abundance of Ne of 2-5\% (all abundances in this paper are given as mass fractions) was derived (Werner \& Wolff 1999).

We present here first results of new observations performed with FUSE and Chandra, whose spectroscopic resolution is more than an order of magnitude better than that of previous FUV and EUV missions (HUT and EUVE). Synthetic spectra were computed with a NLTE code (Werner \& Dreizler 1999) which constructs line blanketed, static, plane-parallel model atmospheres.

\section{FUSE and Chandra spectroscopy}

H 1504+65 was observed with FUSE on Jan. 28, 2002, with an integration time of 10.7 hours. The spectral range covered was $912-1188 \AA$ with a resolution of about $0.1 \AA$. The spectrum is characterized by a few broad photospheric lines of $\mathrm{CIV}$ and $\mathrm{O}$ VI and many narrow interstellar lines, predominantly from $\mathrm{H}_{2}$. Two interesting details are shown in Fig. 1. The left panel is centered around the location of a He II line. It is clearly seen that no He II absorption is detectable 


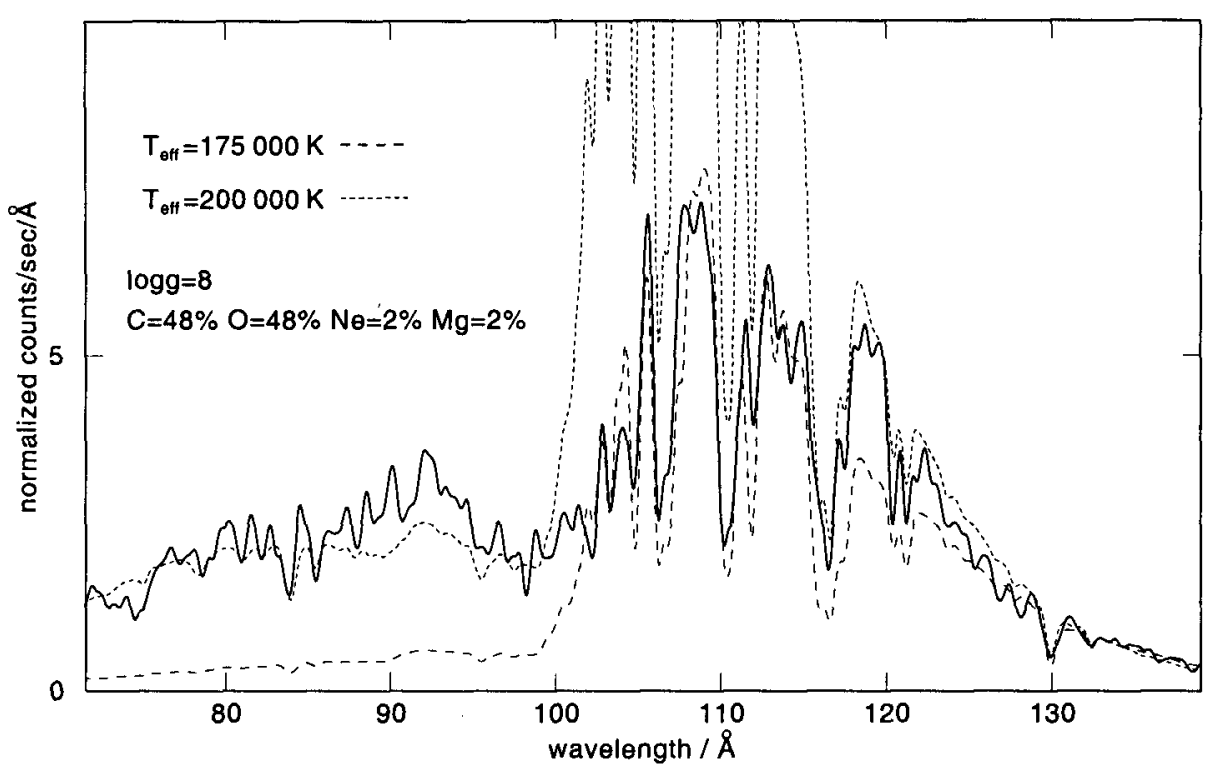

Figure 2. Overview of the Chandra spectrum of $\mathrm{H} 1504+65$ (thick line). Two models with different $T_{\text {eff }}$ are shown. The $175000 \mathrm{~K}$ model fits the overall flux at long wavelengths, while it underestimates the short wavelength flux (dashed line). The hotter model $(200000 \mathrm{~K}$, solid line) fits at short wavelengths but overestimates the long wavelength flux. The model spectra were attenuated by an ISM model with $n_{\mathrm{H}}=$ $8.2 \cdot 10^{19} \mathrm{~cm}^{-2}$ and $9.4 \cdot 10^{19} \mathrm{~cm}^{-2}$, respectively, then folded through the instrument response and normalized to the observation to fit near $130 \AA$. For clarity, all spectra are smoothed with Gaussians $(0.5 \AA$ FWHM $)$.

and a comparison with models shows that the helium abundance is at most of the order of $1 \%$. This confirms the results from a less reliable optical analysis, which was entirely based on the lack of a He II $4686 \AA$ emission line (Werner 1991), and from HUT spectroscopy (Kruk \& Werner 1998). The right panel is centered around the OVI resonance doublet. The observed profile shows no evidence for on-going mass-loss and hence can be fitted with a synthetic profile of a static model. This will allow the derivation of an upper limit for the mass-loss rate from which one can determine the significance of mass-loss for the cooling rate and the surface chemical composition.

H 1504+65 was observed with Chandra on Sep. 27, 2000, with an integration time of 7 hours. Flux was detected in the range $60-160 \AA$ and the spectral resolution is about $0.1 \AA$. Fig. 2 shows the overall spectrum. It is characterized by a roll-off at long wavelengths due to ISM absorption. The maximum flux is detected near $110 \AA$. Between $105 \AA$ and $100 \AA$ the flux drops because of absorption from the OVI edge of the first excited atomic level. The edge is not sharp because of a converging line series and pressure ionization (see Werner \& Wolff, 1999, for detailed model spectra). Below $100 \AA$ the flux decreases, representing 


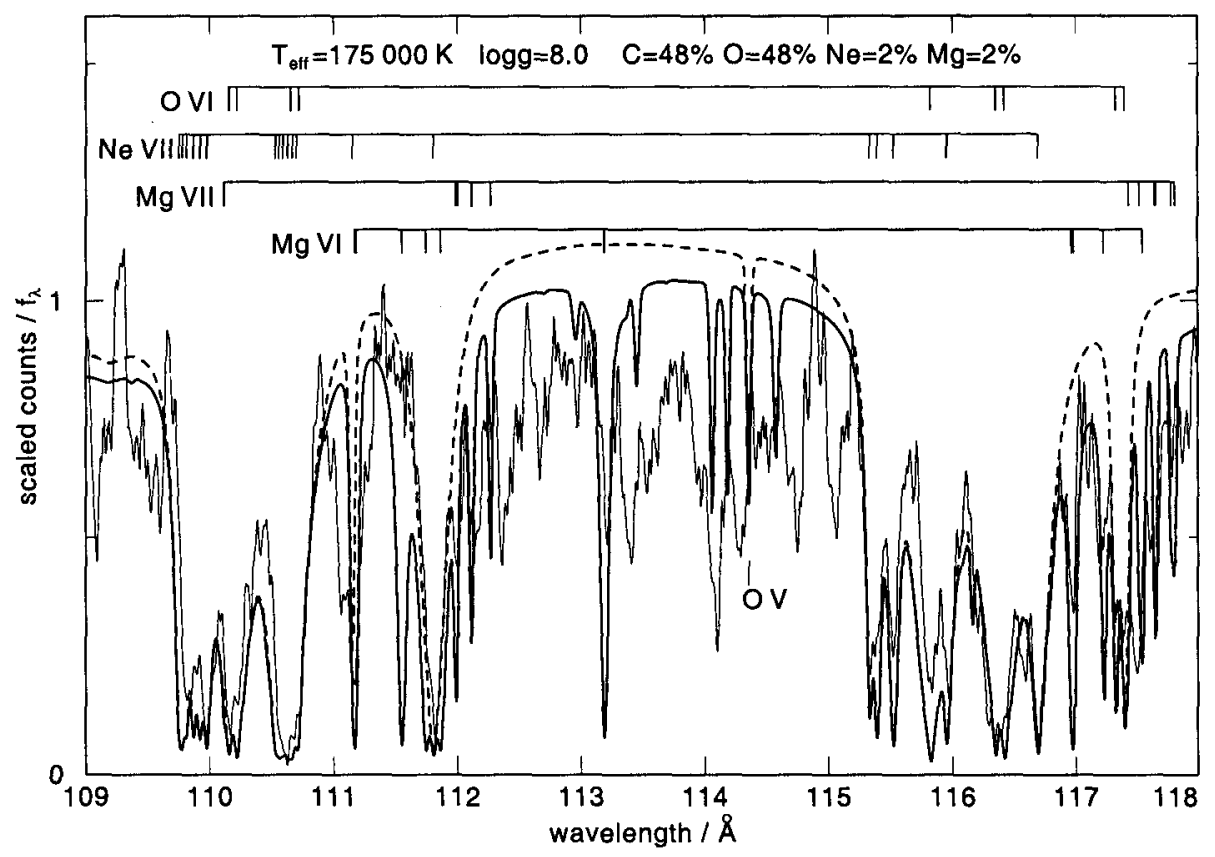

Figure 3. Detail from the Chandra count spectrum of $\mathrm{H} 1504+65$ (thin line), shifted by $-0.06 \AA$ to zero radial velocity. Relative fluxes of two models are overplotted, one including $\mathrm{Mg}$ (solid line) and one without $\mathrm{Mg}$ (dashed line), in order to facilitate the identification of $\mathrm{Mg}$ lines. Observation and model spectra are smoothed with Gaussians (FWHM $0.02 \AA$ and $0.03 \AA$, respectively).

the Wien tail of the photospheric flux distribution. Fig. 2 demonstrates that current models cannot fit the entire wavelength range at a unique temperature, so that $T_{\text {eff }}$ can only be constrained between $175000 \mathrm{~K}$ and $200000 \mathrm{~K}$. At present we favor the higher value, because the excess model flux at $100-120 \AA$ can be explained by missing opacities from iron group elements, as exploratory calculations have shown. Also, a detailed inspection of the relative strengths of Mg VI and $\mathrm{Mg}$ VII lines favors the higher temperature.

Figs. 3 and 4 display astonishing details in the Chandra spectrum. At first sight the spectrum appears to be rather noisy, but comparison with model spectra suggests that most absorption features in the observation, which are not yet fitted with current models, are probably real. Magnesium lines are discovered for the first time in $\mathrm{H} 1504+65$, which however becomes only clear by comparison with modeling with a very detailed $\mathrm{Mg}$ model atom (see in particular Fig. 4, top panel). It comprises more than 120 NLTE levels and 130 individual lines in the Chandra spectral range. It is worth mentioning that fine structure splitting of the metal lines can be detected in the observed spectra down to a separation of only $0.1 \AA$ and requires such detailed modeling. Designing the respective model atoms is cumbersome because atomic data from different sources needs to be 

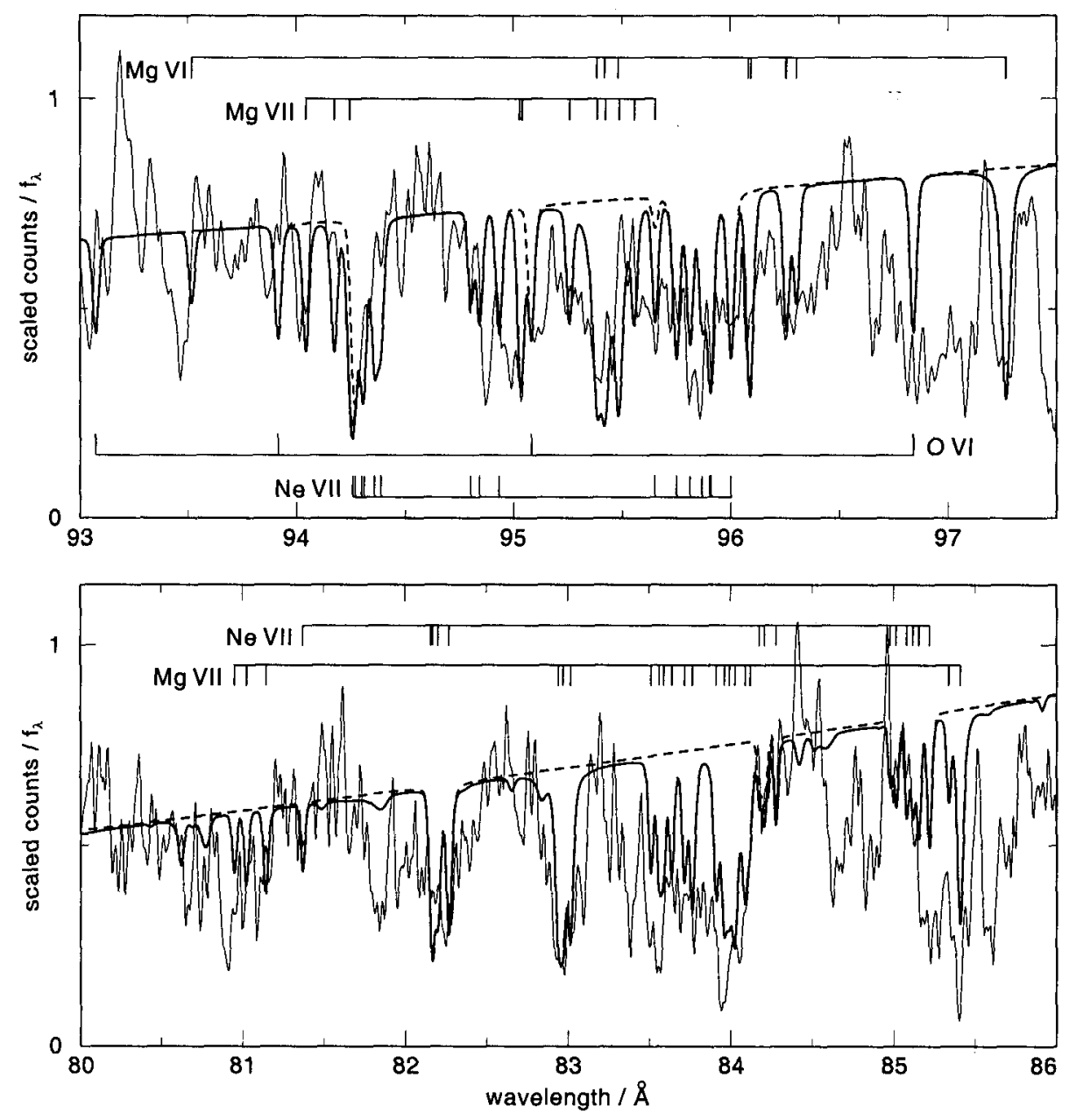

Figure 4. Like Fig. 3, but other spectral regions. All lines included in the models are identified. Additional weak absorption features in the models are due to resonances in the bound-free cross-sections.

collected. Energy levels are obtained from either Bashkin \& Stoner (1975) or the NIST database. Oscillator strengths are taken from TOPBASE, the Opacity Project (OP) database (Seaton et al. 1994). Whenever possible, bound-free cross-sections for atomic levels are also taken from TOPBASE, but in some cases hydrogen-like approximations are necessary. A problem is posed by strong resonances in the OP photoionization data. They are responsible for some strong, absorption line-like features in the computed spectra. Their exact wavelength location is, however, uncertain because the data are results from ab-initio calculations yielding inaccurate energy levels. Collisional rates are computed by usual formulae which employ radiative cross-sections as far as possible. 
As already mentioned, we have computed exploratory models including opacities of heavy metals $(\mathrm{Ca}-\mathrm{Ni})$. This introduces a very large number of additional lines which effectively block flux in the $100-120 \AA$ region and thus may eventually lead to a unique temperature determination. However, identifying individual heavy metal lines will be problematic, because the vast majority of lines in the available lists (Kurucz 1994) have uncertain wavelength positions.

\section{Discussion}

From our first models presented here, the magnesium abundance is of the order $2 \%$, similar to the $\mathrm{Ne}$ abundance determined earlier. This allows two alternative interpretations. Either we see a naked $\mathrm{C} / \mathrm{O}$ white dwarf with $3 \alpha$ processed matter, which is, according to evolutionary models, dominated by $\mathrm{C}$ and $\mathrm{O}$ with an admixture of ${ }^{22} \mathrm{Ne}$ and ${ }^{25} \mathrm{Mg}$ of the order of $1 \%$ (e.g. Iben \& Tutukov 1985). Or we see a naked $\mathrm{O} / \mathrm{Ne} / \mathrm{Mg}$ white dwarf which has undergone carbon burning. A comparison with the respective evolutionary models (Ritossa et al. 1999) suggests that we could look onto such a naked remnant where ${ }^{20} \mathrm{Ne}$ and a mixture of several $\mathrm{Mg}$ isotopes $\left({ }^{24} \mathrm{Mg},{ }^{25} \mathrm{Mg},{ }^{26} \mathrm{Mg}\right)$ should be present. We have already speculated earlier, that the onset of $\mathrm{C}$ burning and subsequent evolutionary processes might explain the uniqueness of $\mathrm{H} 1504+65$. In order to confirm this idea, we will also look for sodium absorption lines, because the stellar models also predict a relatively high amount of ${ }^{23} \mathrm{Na}$ in this case. To conclude, $\mathrm{H} 1504+65$ might have been one of the "heavyweight" intermediate-mass stars $\left(9 \mathrm{M}_{\odot} \leq\right.$ $M \leq 11 \mathrm{M}_{\odot}$ ) which form white dwarfs with electron-degenerate $\mathrm{O}-\mathrm{Ne}-\mathrm{Mg}$ cores resulting from carbon burning. This speculation is corroborated by the fact that $\mathrm{H} 1504+65$ is one of the most massive PG 1159 stars known $\left(0.86 \pm 0.15 \mathrm{M}_{\odot}\right)$.

Acknowledgments. UV and X-ray data analysis in Tübingen is supported by the DLR under grant 50 OR 0201. MAB is supported by the Particle Physics and Astronomy Research Council, UK. JWK is supported by the FUSE project, funded by NASA contract NAS5-32985.

\section{References}

Bashkin, S., \& Stoner, J. O. Jr. 1975, Atomic Energy Levels \& Grotrian Diagrams-Vol. 1: Hydrogen I - Phosphorus XV, (Amsterdam: North Holland)

Iben, I. Jr., \& Tutukov, A. V. 1985, ApJS, 58, 661

Kruk, J. W., \& Werner, K. 1998, ApJ, 502, 858

Kurucz, R. L. 1994, Kurucz CD-ROM Nos. 20-22, (Cambridge: SAO)

Nousek, J. A., et al. 1986, ApJ, 309, 230

Nugent, J. J., et al. 1983, ApJS, 51, 1

Ritossa, C., Garcia-Berro, E., \& Iben, I. Jr. 1999, ApJ, 515, 381

Seaton, M. J., Yan, Y., Mihalas, D., \& Pradhan, A. K. 1994, MNRAS, 266, 805

Werner, K. 1991, A\&A, 251, 147

Werner, K. 2001, Ap\&SS, 275, 27 
Werner, K., \& Dreizler, S. 1999, J. Comp. \& App. Math., 109, 65

Werner, K., \& Wolff, B. 1999, A\&A, 347, L9 